\title{
Impact of Bep or Carboplatin Chemotherapy on Testicular Function and Sperm Nucleus of Subjects with Testicular Germ Cell Tumor
}

\author{
Marco Ghezzi 1,2, Massimiliano Berretta ${ }^{3}$, Alberto Bottacin', Pierfrancesco Palego', \\ Barbara Sartini', Ilaria Cosci', Livio Finos ${ }^{4}$, Riccardo Selice ${ }^{1}$, Carlo Foresta' ${ }^{1}$ and \\ Andrea Garolla ${ }^{1,2 *}$

\begin{abstract}
${ }^{1}$ Unit of Andrology and Reproductive Medicine, Department of Medicine, University of Padova, Padova, Italy, ${ }^{2}$ Istituto Oncologico Veneto - Istituto di Ricovero e Cura a Carattere Scientifico, Padova, Italy, ${ }^{3}$ Department of Medical Oncology, CRO Aviano National Cancer Institute IRCCS, Aviano, Italy, ${ }^{4}$ Department of Statistical Sciences, University of Padova, Padova, Italy
\end{abstract}

\section{OPEN ACCESS}

Edited by: Giovanni Li Volti,

University of Catania, Italy

Reviewed by: Loredana Gandini,

Sapienza University of Rome, Italy Gaetano Facchini, Istituto Nazionale Tumori Fondazione

G. Pascale, Italy

*Correspondence: Andrea Garolla andrea.garolla@unipd.it

Specialty section: This article was submitted to Experimental Pharmacology and Drug

Discovery,

a section of the journal Frontiers in Pharmacology

Received: 04 April 2016 Accepted: 27 April 2016 Published: 13 May 2016

Citation:

Ghezzi M, Berretta M, Bottacin A, Palego P, Sartini B, Coscil, Finos L,

Selice R, Foresta $C$ and Garolla $A$ (2016) Impact of Bep or Carboplatin Chemotherapy on Testicular Function and Sperm Nucleus of Subjects with

Testicular Germ Cell Tumor. Front. Pharmacol. 7:122. doi: 10.3389/fphar.2016.00122
Young males have testicular germ cells tumors (TGCT) as the most common malignancy and its incidence is increasing in several countries. Besides unilateral orchiectomy (UO), the treatment of TGCT may include surveillance, radiotherapy, or chemotherapy (CT), basing on tumor histology and stage of disease. It is well known that both radio and CT may have negative effects on testicular function, affecting spermatogenesis, and sex hormones. Many reports investigated these aspects in patients treated with bleomycin, etoposide, and cisplatin (BEP), after UO. In contrast no data are available on the side effects of carboplatin treatment in these patients. We included in this study 212 consecutive subjects who undergone to sperm banking at our Andrology and Human Reproduction Unit after UO for TGCT. Hundred subjects were further treated with one or more BEP cycles (BEP-group), 54 with carboplatin (CARB group), and 58 were just surveilled (S-group). All patients were evaluated for seminal parameters, sperm aneuploidy, sperm DNA, sex hormones, volume of the residual testis at baseline (TO) and after 12 (T1) and 24 months (T2) from UO or end of CT. Seminal parameters, sperm aneuploidies, DNA status, gonadic hormones, and testicular volume at baseline were not different between groups. At T1, we observed a significant reduction of sperm concentration and sperm count in the BEP group versus baseline and versus both Carb and S-group. A significant increase of sperm aneuploidies was present at T1 in the BEP group. Similarly, the same group at 1 had altered sperm DNA integrity and fragmentation compared with baseline, S-group and Carb group. These alterations were persistent after 2 years from the end of BEP treatment. Despite a slight improvement at T2, the BEP group had still higher percentages of sperm aneuploidies than other groups. No impairment of sperm aneuploidies and DNA status were observed in the Carb group both after 1 and 2 years from the end of treatment. Despite preliminary, these data demonstrate that in selected patients with TGCTs CT with carboplatin represents a therapeutic option that that seems to not affect sex hormones, spermatogenesis, and sperm nucleus.

Keywords: BEP, carboplatin, chemotherapy, fertility, testis cancer 


\section{INTRODUCTION}

Young males aged 15-40 years have testicular germ cells tumors (TGCTs) as the most common malignancy that may affect their health status (Schmoll et al., 2010; Oldenburg et al., 2013).

It has been reported that about 18,000 European subjects over reproductive age develop a TGCTs every year (Znaor et al., 2014) and its incidence is increasing in several countries over the past 50 years (Huyghe et al., 2007). Many risk factors have been studied as a pre-disposing factor in the development of this cancer, but only for some there is a high level of evidence (Garolla et al., 2012; Ferlin and Foresta, 2014). Early diagnosis and modern treatment have resulted in over 95\% survival rate and improved quality of life of testicular cancer survivors (Schagen et al., 2008; Ping et al., 2014).

Usually, treatment for TGCTs is a combination of orchiectomy and radiation therapy (RT), platinum-based chemotherapy (CT) or just surveillance, based on tumor histology and stage of disease. CT including alkylating agents or RT directed to the gonads are usually used for stage II and relapsed diseases. These treatments have well-known negative side effects on testicular functions affecting both spermatogenesis and steroidogenesis up to oligo/azoospermia and hypogonadism. Moreover a negative impact of cancer treatments on sperm DNA and chromosomes has been shown after years from the end of therapy (Tempest et al., 2008; O’Flaherty et al., 2010; Patassini et al., 2013). It was proposed that, between CT drugs, etoposide appears to be a good candidate for aneuploidy sperm induction as it interacts with topoisomerase II during anaphase I and II of meiosis (De Mas et al., 2001). Besides the alteration of spermatogenesis, bleomycin, etoposide and cisplatin (BEP)-use has been reported to affect sperm chromosome and DNA status (De Mas et al., 2001). These authors, investigating sperm cell aneuploidy for chromosomes 7 , $16,18, \mathrm{X}$, and $\mathrm{Y}$ in a cohort of BEP-treated TGCTs described a significant increase in the frequency of diploidy and disomy for these chromosomes compared to healthy controls. In a subsequent study done in a population of TGCTs patients, Tempest et al. (2008) documented an increase of aneuploidies for sperm chromosomes, X, Y, 13, and 21, 6 months after the end of 2-4 BEP cycles that decline to pretreatment levels only at 18 months after the end of treatment. Based on these findings, some authors suggested that treated patients should be counseled to avoid conceptions up to 2 years after the end of treatments (Hassold et al., 1996; Tempest et al., 2008).

Exposure to the treatment regimen for testis cancer (BEP or cyclophosphamide) causes DNA damage, inducing crosslinks as well as single and double strand breaks: therefore these treatments may represent a potential risk to subsequent generations if DNA and telomere damage in sperm are sustained; moreover it was reported that significant sperm DNA damage and reduced chromatin compaction persisted in men for up to 24 months after BEP treatment (Zhou and Bartek, 2004; O'Flaherty et al., 2010; Liu et al., 2015). Recently Liu et al. (2015) demonstrated that in vivo exposure of rat male germ cells to a BEP regimens, analogous to that given to testis cancer patients, induces telomere shortening in all stages of spermatogenesis raising concern about the potential risk of BEP exposure to subsequent generations. Interestingly DNA double strand breaks were increased significantly in zygotene rat male germ cells exposed to BEP regimens, as assessed by gammaH2AX immunofluorescence (Liu et al., 2015). Furthermore a marked increase in DNA Fragmentation Index after 3 and 6 months to the end of BEP treatment was demonstrated in a series of TGCTs patients, improving after 12 and 24 months to the end of treatments (Paoli et al., 2015).

On this basis, patients candidate to $\mathrm{CT}$ usually receive medical counseling about sperm preservation, endocrine followup and genetic counseling to avoid conceptions during the treatments period up to 2 years after the end of therapy aimed to avoid possible defective genome transmission to their offspring (Tempest et al., 2008; Ping et al., 2014). Despite some authors raised concern about the hypothesis that spermatozoa may carry damages DNA even long after treatments have finished, there is no actual evidence to suggest that previous TGCTs treatments are associated with birth defect (Thomson et al., 2002; Ping et al., 2014).

Because most patients and their parents are interested to preserve fertility, sperm banking should be recommended before cancer treatments (Jeruss and Woodruff, 2009). Although sperm cryopreservation allow cancer survivors to reach fertility with in vitro fertilization (IVF), new treatment strategies with a lower impact on testicular function are challenging to preserve spontaneous fertility and to reduce sperm DNA alterations and hormones impairments avoiding potential hypogonadism (Choy and Brannigan, 2013). Many reports have investigated the impact on testicular function and in particular on spermatogenesis of RT and CT with BEP in men before and after orchiectomy (Thomson et al., 2002; Bujan et al., 2013; Choy and Brannigan, 2013). In contrast no data are available on the effects of a carboplatin (CARB)-based CT on these topics and, in particular, no data are available on the comparison between BEP-treated and CARBtreated subjects.

Aim of the present study was to assess the impact of orchiectomy followed by CT (BEP treatment, or surveillance alone on sperm parameters, sperm aneuploidies and DNA fragmentation, sex hormones, and testicular volume in TGCTs cancer patients at basal and at 12 and 24 months after the end of any treatment. In particular, we aimed to evaluate the possible gonadotoxic effects of CARB in comparison with surveillance and BEP treatments.

\section{MATERIALS AND METHODS}

\section{Subjects}

This retrospective study enrolled 212 consecutively subjects who had been sent by oncologist to Unit of Andrology and Reproductive Medicine of Padua for sperm banking after unilateral orchiectomy (UO) for TGCTs. Patients had a cancer stage ranging from first to third. The subjects were divided into three groups based on the management of disease suggested by the oncologist: 100 with one to four BEP cycles based on tumor histology, 54 with CARB one cycle (group 3), and 58 had no treatment (surveillance alone). Patients were evaluated at baseline 
(T0) and then followed-up after 12 and 24 months from the start of treatments ( $\mathrm{T} 1$ and $\mathrm{T} 2$, respectively) for sperm parameters, sex hormones, testicular volume, sperm aneuploidies, and DNA integrity. We have excluded from the study patients with know causes of infertility such as Klinefelter syndrome, Y chromosome microdeletions, varicocele in the remaining testis and history of orchitis or cryptorchidism. Written informed consent was obtained from all patients, and the study protocol was approved by the local ethics committee.

\section{Semen Sample Collection and Processing}

Semen samples were obtained by masturbation after 2-5 days of sexual abstinence. After liquefaction at room temperature, semen volume, $\mathrm{pH}$, sperm concentration, total sperm count (TSC), viability, motility, and normal morphology were determined according to World Health Organization guidelines for semen analysis (World Health Organization [WHO], 1999). Semen samples were then washed three times with sterile phosphatebuffered saline (PBS 1), and the pellet was used for the subsequent analyses.

\section{Hormone Assay}

Blood sample was collected in the fasting state between 08.00 and 1000 h. Serum FSH, LH, and total testosterone were evaluated by commercial electrochemiluminescence immunoassay methods (Elecsys, 2010; Roche Diagnostics, Mannheim, Germany) as reported elsewhere (Selice et al., 2011). For all parameters, the intra- and inter-assay coefficients of variation were $<8$ and $10 \%$, respectively).

\section{Ultrasound (US) Scanning}

To evaluate testicular size, morphology and normal tissue echo pattern, all subjects were studied with ultrasonography. All US examinations were made using a high resolution machine (Aplio XV Toshiba, Tokyo, Japan) equipped with a 7-13 MHz multifrequency linear probe by the same experienced doctor (P.P.). Intra-observer variability for testicular volume was estimated to be less than $10 \%$ as reported in a previous study of our group (Selice et al., 2011).

\section{FISH Analysis for Sperm Aneuploidy}

The study of sperm aneuploidy was performed by multicolor FISH, as reported elsewhere (Garolla et al., 2013). The DNA hybridization was performed using a human satellite probe-specific mix for chromosomes X, Y, and 18 (Kreatech Diagnostics). Probes were direct-labeled with fluorochrome PlatinumBright495 for chromosome $\mathrm{X}$, resulting in a green signal and fluorochrome PlatinumBright550 for chromosome $\mathrm{Y}$, resulting in a red signal, for the detection of chromosome 18 a PlatinumBright415 direct-labeled specific probe was used, resulting in a blue signal.

The DNA denaturation of sperm and probes, incubation, posthybridization washing, and nuclear staining were performed according to the Kreatech protocol. After preparation, slides were observed using a fluorescence microscope (Nikon Eclipse
E600) equipped with a triple band-pass filter set [fluorescein isothiocyanate (FITC), tetrarhodamine isothiocyanate (TRITC), 6-diamino-2-phenylindole (DAPI)]. Single spots were evaluated as reported elsewhere (Robbins et al., 1995). For each patient, at least 2,500 cells were scored.

\section{Acridine Orange Test (AO Test)}

The AO test was used to assess the heterogeneity of sperm DNA. Briefly $10 \mu \mathrm{l}$ of semen sample and equal volume of AO solution $[10 \mathrm{~mL}$ acridine orange stain (1\%) was mixed with $2.5 \mathrm{~mL} \mathrm{Na} 2 \mathrm{HPO} 4.7 \mathrm{H} 2 \mathrm{O}(0.3 \mathrm{~mol} / \mathrm{l})$, and $40 \mathrm{~mL}$ citric acid $(0.1 \mathrm{~mol} / \mathrm{l})]$ were mixed together on the surface of a glass slide and covered with a glass coverslip. The sample was then evaluated with a fluorescence microscope with a $490 \mathrm{~nm}$ excitation light and $530 \mathrm{~nm}$ barrier filter. Nuclei from 200 spermatozoa were examined and scored as with green- or red-fluorescence. When the head showed green fluorescence, sperm were considered normal (double-stranded DNA); they were considered denatured when fluorescence was red (single-stranded DNA). Results were expressed as the percentage of sperm that showed altered (red) fluorescence (Eggert-Kruse et al., 1996).

\section{TUNEL Assay}

The DNA fragmentation was evaluated by TUNEL assay performed with the Cell Death Detection Kit (Roche Diagnostics). Fluorescein isothiocyanate-deoxyuridine triphosphate was used as label according to the manufacturer's instructions and counterstained with 40,6-diamidino-2phenylindole. Briefly, after preparation, sperm samples were smeared on microscope slides, fixed with $4 \%$ paraformaldehyde, and permeabilized with $0.1 \%$ Triton $\mathrm{X}-100$ in $0.1 \%$ sodium citrate. The specimens were incubated in TUNEL reaction mixture in a humidified atmosphere for $60 \mathrm{~min}$ at $37^{\circ} \mathrm{C}$ in the dark. The slides were rinsed twice, and then $1 \mathrm{~mL} 40,6-$ diamidino-2-phenylindole was added to counterstain the cells. Four hundred spermatozoa were analyzed at 40 by using a Nikon E600 epifluorescence light microscope equipped with a 450-490-nm excitation (Nikon), and those cells with green fluorescence were considered to be altered.

\section{$\gamma \mathrm{H} 2 \mathrm{AX}$ Flow-Cytometric Analysis}

The sperm cells were fixed, permeabilized, washed in PBS and centrifuged as reported in the previous paragraph. Thereafter, pellets were resuspended in $200 \mu \mathrm{l}$ of PBS-0.1\% BSA and equally split in two vials used as samples and negative controls. The staining for $\gamma \mathrm{H} 2 \mathrm{AX}$ was conducted resuspending the pellet samples in $200 \mu \mathrm{l}$ of polyclonal rabbit anti- $\gamma \mathrm{H} 2 \mathrm{AX}$ antibody (1:200 dilution), (Millipore, Billerica, MA, USA) for $90 \mathrm{~min}$ at $37^{\circ} \mathrm{C}$. Negative controls and samples were then rinsed in PBS$0.1 \%$ BSA solution and after centrifugation were resuspended in $200 \mu \mathrm{l}$ of secondary goat anti-rabbit IgG-fluorescein conjugated antibody (1:400 dilutions; Millipore, Billerica, MA, USA) for $45 \mathrm{~min}$ at $37^{\circ} \mathrm{C}$. Finally, the sperm cells were rinsed once in PBS-0.01\% tween-20 solution (Sigma-Aldrich, St. Louise, MO, USA) for $5 \mathrm{~min}$ at room temperature and centrifuged. Pellet was washed once in a PBS solution for $5 \mathrm{~min}$ at room temperature and centrifuged. Before analysis with a Flow Cytometer cells were 
resuspended in $300 \mu \mathrm{l}$ of Propidie Iodide solution $(25 \mathrm{ng} / \mathrm{m}$ of $\mathrm{PI}$ in $\mathrm{H}_{2} \mathrm{O}$; Sigma-Aldrich, St. Louise, MO, USA). Samples were then analyzed on a FACScan flow cytometer (Becton Dickinson, Mountain View, CA, USA) equipped with a $15 \mathrm{~mW}-488 \mathrm{~nm}$, air cooled, argon ion laser for excitation. For each sample, 40000 events were recorded within the characteristic flameshaped region in the forward scatter/side scatter (FSC/SSC) dot plot. Data were acquired and analyzed with Cellquest analysis software. The average amount of $\gamma \mathrm{H} 2 \mathrm{AX}$ antibody staining relative to the untreated control was scored as the percentage of spermatozoa having fluorescence intensities above a threshold excluding $\leq 1 \%$ of the events in the negative sample (Li et al., 2006). Results were expressed as percentage of DSBs in total sperm analyzed.

\section{Statistical Analysis}

Results are expressed as mean \pm SD. Variations in semen and hormonal parameters and testicular volume were considered and are expressed as raw differences.

Comparisons among four groups were performed through ANOVA test. To evaluate effects of therapy along time, twosample $t$-tests were computed using T0 as reference time point. To compare therapies, also considering the initial individual characteristics, a repeated measures ANOVA is performed for each parameter of interest. The software package $R$ ( $R$ Core Team, 2012) was used for statistical analyses. Probability values of $<0.05$ were considered statistically significant.

\section{RESULTS}

Mean age of different groups was not significantly different $(S=32.0 \pm 1.4, \mathrm{CARB}=34.2 \pm 3.5, \mathrm{BEP}=34.0 \pm 2.8$ years $)$.

In Table 1 are reported seminal parameters, hormonal levels, and testicular volume at different time points of the study of surveilled patients $(S)$ and patients treated with CARB or BEP observed at different time points of the study. At baseline, seminal parameters, hormonal status, and testicular volume did not differ between three groups. At T1 the BEP group had a significant reduction of sperm concentration and sperm count compared with baseline $(p<0.0001)$ and versus both CARB and S-group $(p<0.0001)$. At T2, these semen alterations were still evident in BEP patients compared with other groups. All groups showed no significant variations in sperm motility and morphology during the whole study. After orchiectomy, all groups showed a progressive increase of FSH plasma levels. In treated patients (both CARB and BEP groups), this change was already significant at T1 $(p<0.001$ versus T0) while $S$-group showed a significant increase only at $\mathrm{T} 2$. In contrast, $\mathrm{LH}$, total testosterone, and testicular volume remained unchanged in all groups.

Table 2 shows results of FISH test to evaluate sperm aneuploidies (autosomes, sex chromosomes, and total aneuploidies), acridine orange, TUNEL and $\gamma \mathrm{H} 2 \mathrm{AX}$ tests to evaluate, respectively, DNA compaction, single- or doublestrand fragmentation and double-strand fragmentation. No differences between groups were observed at baseline. When, we considered FISH analysis for autosome and sex chromosomes

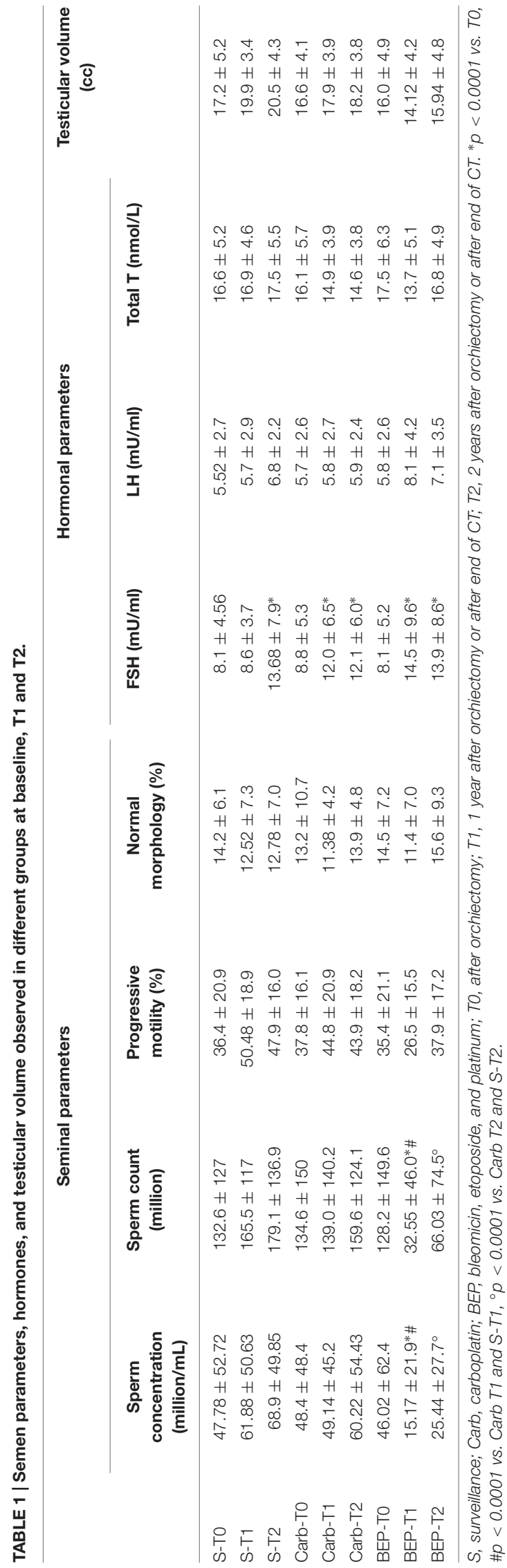


TABLE 2 | Sperm aneuploidies and nuclear status observed in different groups at baseline, T1 and T2.

\begin{tabular}{|c|c|c|c|c|c|c|}
\hline & FISH (Aut.) (\%) & FISH (Sex) (\%) & FISH (Tot.) (\%) & Acridine orange (\%) & TUNEL (\%) & $\gamma \mathrm{H} 2 \mathrm{AX}(\%)$ \\
\hline S-TO & $0.61 \pm 0.32$ & $0.73 \pm 0.52$ & $1.34 \pm 0.76$ & $19.78 \pm 4.0$ & $16.13 \pm 4.16$ & $5.35 \pm 3.35$ \\
\hline S-T1 & $0.64 \pm 0.27$ & $0.68 \pm 0.28$ & $1.33 \pm 0.47$ & $19.52 \pm 3.59$ & $15.74 \pm 4.36$ & $6.04 \pm 3.3$ \\
\hline S-T2 & $0.65 \pm 0.29$ & $0.65 \pm 0.30$ & $1.29 \pm 0.44$ & $18.87 \pm 4.05$ & $15.09 \pm 4.66$ & $6.13 \pm 3.14$ \\
\hline Carb-TO & $0.69 \pm 0.26$ & $0.72 \pm 0.36$ & $1.41 \pm 0.50$ & $20.21 \pm 4.4$ & $16.38 \pm 3.88$ & $6.12 \pm 2.88$ \\
\hline Carb-T1 & $0.69 \pm 0.19$ & $0.61 \pm 0.24$ & $1.30 \pm 0.35$ & $18.83 \pm 4.65$ & $16.21 \pm 4.1$ & $6.79 \pm 3.93$ \\
\hline Carb-T2 & $0.69 \pm 0.22$ & $0.60 \pm 0.27$ & $1.29 \pm 0.36$ & $17.29 \pm 4.68$ & $16.0 \pm 4.53$ & $6.58 \pm 3.75$ \\
\hline ВEP-TO & $0.69 \pm 0.35$ & $0.8 \pm 0.4$ & $1.49 \pm 0.68$ & $20.18 \pm 5.64$ & $16.94 \pm 5.22$ & $6.97 \pm 4.58$ \\
\hline BEP-T1 & $1.08 \pm 0.61$ & $1.19 \pm 0.78$ & $2.27 \pm 1.38^{* \#}$ & $25.43 \pm 6.17^{* \#}$ & $21.49 \pm 4.94^{* \#}$ & $11.42 \pm 4.28^{* \#}$ \\
\hline BEP-T2 & $0.83 \pm 0.41$ & $0.88 \pm 0.54$ & $1.9 \pm 0.89^{\circ} \$$ & $22.42 \pm 5.02 * \$$ & $20.64 \pm 5.2^{* \#}$ & $10.09 \pm 3.34^{* *}$ \\
\hline
\end{tabular}

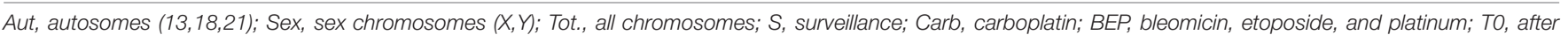

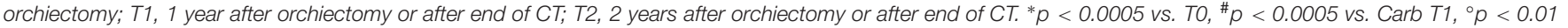
vs. T0, $\$ p<0.01$ vs. carb T2, ** $p<0.05$ vs. T0 and Carb T2.

separately data were not different in all groups along the study. However, considering autosomes and sex chromosomes as a whole, BEP subjects showed significantly higher sperm aneuploidies at T1 ( $p<0.01$ vs. T0 and CARB- group). This alteration was slightly reduced at $\mathrm{T} 2$, even if the aneuploidy rate in BEP group was still significantly higher than in other groups $(p<0.05)$. Similarly to sperm aneuploidies, at T1 sperm DNA compaction and fragmentation (both single- and double strand breaks) of BEP group were significantly impaired compared to baseline, S- and CARB group. These alterations were persistent after 2 years from the end of BEP treatment. No impairments of sperm aneuploidies, DNA compaction and fragmentation were observed in the CARB group both after 1 and 2 years from the end of treatment. In this study, we found no factor able to predict changes of sperm parameters, sperm nucleus, and hormones in the response to $\mathrm{CT}$.

In Figure $\mathbf{1}$ are reported some examples of different tests performed to evaluate sperm nuclear status in surveillance and CT treated patients. Figures $\mathbf{1 c}, \mathbf{d}$ and thin lines of Figures $\mathbf{1 e , f}$ show examples of sperm from BEP patients.

\section{DISCUSSION}

Testicular cancer is the most frequent malignancy affecting males at reproductive age. It has been reported that a significant number of European subjects over reproductive age develop a TGCTs every year (Znaor et al., 2014) and its incidence is increasing in several countries over the past 50 years (Huyghe et al., 2007). Actually the new therapeutic strategies and follow-up allow survivorship in over $95 \%$ of cases. After inguinal orchiectomy patients can be treated by RT on pelvic lymph nodes, CT or simply surveilled on the basis of tumor histology, staging, prognostic factors, and patients decision after oncological counseling. Combined therapy with etoposide, cisplatin, and bleomycin is largely considered a useful and effective option for the treatment of TGCTs (Oldenburg et al., 2013).

In 2005 it has been introduced the possibility of treatment with CARB alone in selected cases of TGCTs (seminomas and first stage of disease). Moreover, it has been demonstrated the non-inferiority of such treatment compared with RT, especially

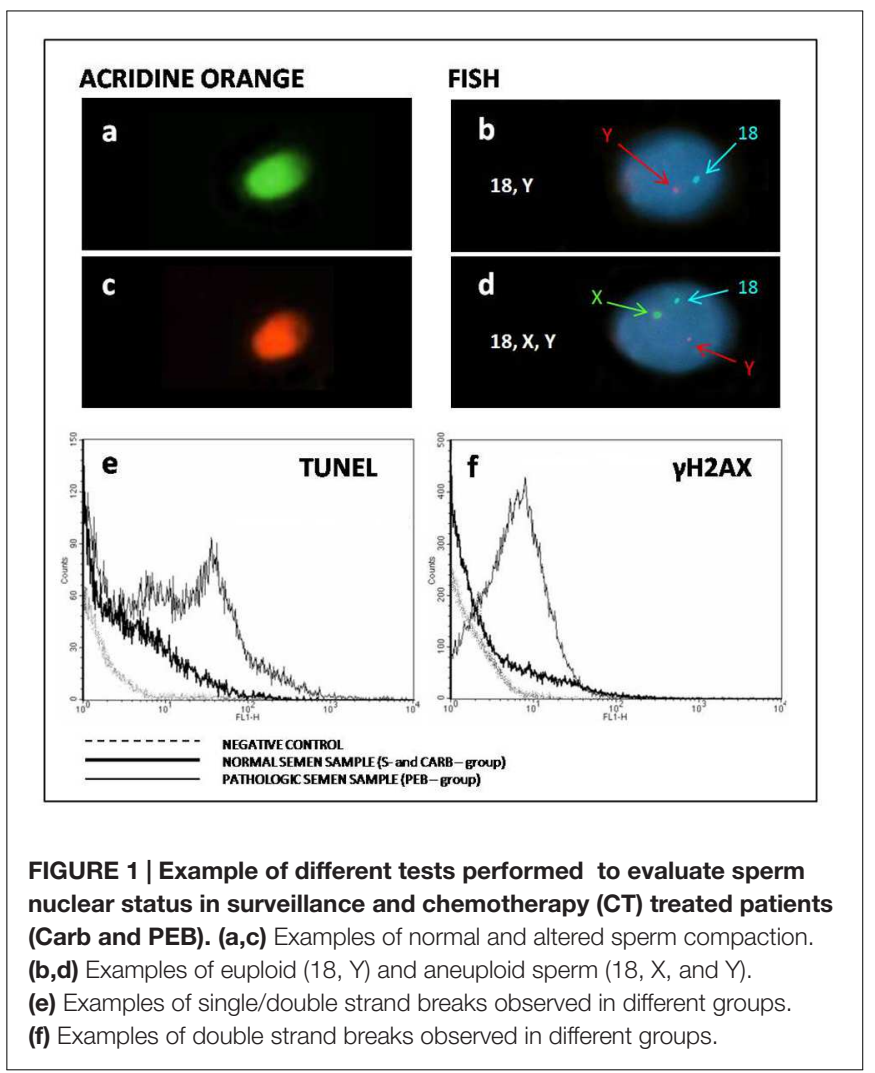

with regard to the disease-free period (Oliver et al., 2005). The risk of second malignancy like non-testicular germ cell has been associated with moderate-dose infradiaphragmatic RT in stage I seminoma. Further data by a subsequent study have later confirmed these findings (Oliver et al., 2011) and were followed by a broad scientific debate on the effectiveness of one cycle of CARB compared with RT. In particular, the matter of debate was the methodology to study renal function for decisions about CARB dose (Dieckmann et al., 2011; Stenning et al., 2011). Recently, this aspect has been definitely clarified and the gold standard for GFR measurement and consequently dose calculation of adjuvant CARB CT is considered (51)Cr-EDTA 
(Quinton et al., 2013). Based on this evidence, the number of people suffering from TGCTs treated with one cycle CARB alone has increased in recent years.

It is far known that conventional CT regimens used in TGCTs patients as BEP, may affect male fertility and endocrine function of the residual testis (Hassold et al., 1996; Tempest et al., 2008; Bujan et al., 2013). Moreover, BEP use has been reported to affect sperm chromosomes and to alter DNA status (De Mas et al., 2001; Zhou and Bartek, 2004; Liu et al., 2015; Paoli et al., 2015). In contrast, no data are available regarding the impact of treatment with CARB on gonadal function. In this preliminary study, we evaluated for the first time the effect of one cycle CARB on semen parameters, sex hormones, sperm aneuploidies, and DNA status in a group of TGCTs patients treated with BEP or one cycle of CARB followed at 12 (T1) and 24 months (T2) after therapy. A third group of TGCTs patients in which oncologist suggested only surveillance after orchiectomy, served as a control group ( $S$-group). Our results confirmed that BEP treatment has a detrimental effect on sperm parameters. In contrast, therapy with CARB alone seems to not affect semen quality. In fact, as in surveillance patients, semen parameters, hormones, and volume of the residual testis remained unchanged. Moreover, our findings showed an increase of sperm aneuploidies after BEP-therapy and these alterations persisted upon 24 months after the end of treatment. Moreover, we observed negative effects of BEP therapy on sperm DNA integrity, persisting upon 24 months from the end of treatments. In our experience, CARB use did not affect sperm aneuploidies, DNA compaction, and DNA fragmentation

\section{REFERENCES}

Berretta, M., Di Francia, R., and Tirelli, U. (2014). Editorial - The new oncologic challenges in the 3RD millennium. W.C.R.J. 1:e133.

Bujan, L., Walschaerts, M., Moinard, N., Hennebicq, S., Saias, J., Brugnon, F., et al. (2013). Impact of chemotherapy and radiotherapy for testicular germ cell tumors on spermatogenesis and sperm DNA: a multicenter prospective study from the CECOS network. Fertil. Steril. 100, 673-680. doi: 10.1016/j.fertnstert.2013.05.018

Choy, J. T., and Brannigan, R. E. (2013). The determination of reproductive safety in men during and after cancer treatment. Fertil. Steril. 100, 1187-1191. doi: 10.1016/j.fertnstert.2013.05.018

De Mas, P., Daudin, M., Vincent, M. C., Bourrouillou, G., Calvas, P., Mieusset, R., et al. (2001). Increased aneuploidy in spermatozoa from testicular tumour patients after chemotherapy with cisplatin, etoposide and bleomycin. Hum. Reprod. 16, 1204-1208. doi: 10.1093/humrep/16.6.1204

Dieckmann, K. P., Matthies, C., and Kliesch, S. (2011). Carboplatin does not prevent contralateral testicular tumors in patients with seminoma. J. Clin. Oncol. 29, 2944-2945. doi: 10.1200/JCO.2011.35.7970

Eggert-Kruse, W., Rohr, G., Kerbel, H., Schwalbach, B., Demirakca, T., Klinga, K., et al. (1996). The Acridine Orange test: a clinically relevant screening method for sperm quality during infertility investigation? Hum. Reprod. 11, 784-789. doi: 10.1093/oxfordjournals.humrep.a019255

Ferlin, A., and Foresta, C. (2014). Testis cancer: genes, environment, hormones. Front. Endocrinol. (Lausanne) 5:172. doi: 10.3389/fendo.2014.00172

Garolla, A., Pizzol, D., Bertoldo, A., De Toni, L., Barzon, L., and Foresta, C. (2013). Association, prevalence and clearance of human papillomavirus and antisperm antibodies in infected semen samples from infertile patients. Fertil. Steril. 99, 125-131. doi: 10.1016/j.fertnstert.2012.09.006

Garolla, A., Pizzol, D., Bertoldo, A., Ghezzi, M., Carraro, U., Ferlin, A., et al. (2012). Testicular cancer and HPV semen infection. Front. Endocrinol. (Lausanne) 3:172. doi: $10.3389 /$ fendo. 2012.00172 at any time of the study. Basing also on these results and the risk of second tumors (non-TGCT0), in patients treated with RT, the use of CARB mono-CT is suggested in selected patients (tumor histology, stage of disease, and prognostic factors) with TGCTs.

\section{CONCLUSION}

Our results although preliminary, denote that CARB alone seems not to have a negative effect on fertility and sex hormones in selected TGCTs patients. Despite the small amount of patients and the need of larger studies to confirm our data, the present study is the first on this topic suggesting that one cycle CARB, besides representing a valid therapeutic option, seem to not affect spermatogenesis, sperm aneuploidies, DNA integrity, and gonadal hormones in TGCTs orchidectomized patients. Moreover, the multidisciplinary approach is mandatory to choose the best treatment in patients with cancer disease potentially curable (Berretta et al., 2014).

\section{AUTHOR CONTRIBUTIONS}

Selected the patients: MG, MB, AG, PP, and RS. Conceived and designed the experiments: MG, AG, and CF. Performed the experiments: AB, PP, BS, and IC. Analyzed the data: MG, LF, and AG. Contributed reagents/materials/analysis tools: AB, IC, BS, and PP. Wrote and revised the manuscript: MG, MB, CF, and AG.

Hassold, T., Abruzzo, M., Adkins, K., Griffin, D., Merrill, M., Millie, E., et al. (1996). Human aneuploidy : incidence, origin and etiology. Environ. Mol. Mutagen 28, 167-175. doi: 10.1002/(SICI)1098-2280(1996)28

Huyghe, E., Plante, P., and Thonneau, P. F. (2007). Testicular cancer variations in time and space in Europe. Eur. Urol. 51, 621-628. doi: 10.1016/j.eururo.2006.08.024

Jeruss, J. S., and Woodruff, T. K. (2009). Preservation of fertility in patients with cancer. N. Engl. J. Med. 360, 902-911. doi: 10.1056/NEJMra0801454

Li, Z., Yang, J., and Huang, H. (2006). Oxidative stress induces H2AX phosphorylation in human spermatozoa. FEBS Lett. 580, 6161-6168. doi: 10.1016/j.febslet.2006.10.016

Liu, M., Maselli, J., Hales, B. F., and Robaire, B. (2015). The effects of chemotherapy with bleomycin, etoposide and cis-platinum on telomeres in rat male germ cells. Andrology 3, 1104-1112. doi: 10.1111/andr.12102

O'Flaherty, C., Hales, B. F., Chan, P., and Robaire, B. (2010). Impact of chemotherapeutics and advanced testicular cancer or Hodgkin lymphoma on sperm deoxyribonucleic acid integrity. Fertil. Steril. 94, 1374-1379. doi: 10.1016/j.fertnstert.2009.05.068

Oldenburg, J., Fossa, S. D., Nuver, J., Heidenreich, A., Schmoll, H. J., Bokemeyer, C., et al. (2013). Testicular seminoma and non-seminoma: ESMO clinical practice guidelines for diagnosis, treatment and follow-up. Ann. Oncol. 24(Suppl. 6), 125-132. doi: 10.1093/annonc/mdt304

Oliver, R. T., Mason, M. D., Mead, G. M., von der Maase, H., Rustin, G. J., Joffe, J. K., et al. (2005). Radiotherapy versus singledose carboplatin in adjuvant treatment of stage I seminoma: a randomised trial. Lancet 366, 293-300. doi: 10.1016/S0140-6736(05) 66984-X

Oliver, R. T., Mead, G. M., Rustin, G. J., Joffe, J. K., Aass, N., Coleman, R., et al. (2011). Randomized trial of carboplatin versus radiotherapy for stage I seminoma: mature results on relapse and contralateral testis cancer rates in MRC TE 19/EORTC 30982 study. J. Clin. Oncol. 29, 957-962. doi: 10.1200/JCO.2009.26.4655 
Paoli, D., Gallo, M., Rizzo, F., Spanò, M., Leter, G., Lombardo, F., et al. (2015). Testicular cancer and sperm DNA damage: short- and long-term effects of antineoplastic treatment. Andrology 3, 122-128. doi: 10.1111/j.20472927.2014.00250.x

Patassini, C., Garolla, A., Bottacin, A., Menegazzo, M., Speltra, E., Foresta, C., et al. (2013). Molecular karyotyping of human single sperm by array-comparative genomic hybridization. PLOS ONE 8:922. doi: 10.1371/journal.pone.006 0922

Ping, P., Gu, B. H., Li, P., Huang, Y. R., and Li, Z. (2014). Fertility outcome of patients with testicular tumor: before and after treatment. Asian J. Androl. 16, 107-111. doi: 10.4103/1008-682X.122194

Quinton, A., Lewis, P., Ali, P., Morgan, C., and Bertelli, G. (2013). A comparison of measured and estimated glomerular filtration rate for carboplatin dose calculation in stage I testicular seminoma. Med. Oncol. 30:661. doi: 10.1007/s12032-013-0661-1

Robbins, W. A., Baulch, J. E., Moore, D. II, Weyer, H. U., Blakey, D., and Wyrobek, A. J. (1995). Three-probe fluorescent in-situ hybridization to assess chromosome X, Y and 8 aneuploidy in sperm of 14 men from two healthy groups: evidence for a paternal age effect on sperm aneuploidy. Reprod. Fertil. Dev. 7, 799-809. doi: 10.1071/RD9950799

Schagen, S. B., Boogerd, W., Muller, M. J., Bokkel Huinink, W. T., Moonen, L., Meinhardt, W., et al. (2008). Cognitive complaints and cognitive impairment following BEP chemotherapy in patients with testicular cancer. Acta Oncol. 47, 63-70. doi: 10.1080/02841860701518058

Schmoll, H. J., Jordan, K., Huddart, R., Laguna Pes, M. P., Horwich, A., Fizazi, K., et al. (2010). Testicular non seminoma: ESMO Clinical practice guidelines for diagnosis, treatment and follow-up. Ann. Oncol. 21(Suppl. 5), 147-154. doi: 10.1093/annonc/mdq177

Selice, R., Ferlin, A., Garolla, A., Caretta, N., and Foresta, C. (2011). Effects of endogenous FSH on normal human spermatogenesis in adults. Int. J. Androl. 34, e511-e517. doi: 10.1111/j.1365-2605.2010. 01134.x
Stenning, S., Oliver, T., Mead, B., and Gabe, R. (2011). Carboplatin in clinical stage I seminoma: a valuable option for patient management. J. Clin. Oncol. 29, 4210-4211. doi: 10.1200/JCO.2011.36.2434

Tempest, H. G., Ko, E., Chan, P., Robaire, B., Rademaker, A., and Martin, R. H. (2008). Sperm aneuploidy frequencies analysed before and after chemotherapy in testicular cancer and Hodgkin's lymphoma patients. Hum. Reprod. 23, 251-258. doi: 10.1093/humrep/dem389

Thomson, A. B., Campbell, A. J., Irvine, D. S., Anderson, R. A., Kelnar, C. J. H., Hamish, W., et al. (2002). Semen quality and spermatozoal DNA integrity in survivors of childhood cancer: a case-control study. Lancet 360, 361-367. doi: 10.1016/S0140-6736(02)09606-X

World Health Organization [WHO] (1999). WHO Laboratory Manual for the Examination of Human Semen, and Sperm-Cervical Mucus Interaction. Cambridge: Cambridge University Press.

Zhou, B. B., and Bartek, J. (2004). Targeting the checkpoint kinases: chemosensitization versus chemoprotection. Nat. Rev. Cancer 4, 216-225. doi: 10.1038/nrc1296

Znaor, A., Lortet-Tieulent, J., Jemal, A., and Bray, F. (2014). International variations and trends in testicular cancer incidence and mortality. Eur. Urol. 65, 1095-1106. doi: 10.1016/j.eururo.2013.11.004

Conflict of Interest Statement: The authors declare that the research was conducted in the absence of any commercial or financial relationships that could be construed as a potential conflict of interest.

Copyright (C) 2016 Ghezzi, Berretta, Bottacin, Palego, Sartini, Cosci, Finos, Selice, Foresta and Garolla. This is an open-access article distributed under the terms of the Creative Commons Attribution License (CC BY). The use, distribution or reproduction in other forums is permitted, provided the original author(s) or licensor are credited and that the original publication in this journal is cited, in accordance with accepted academic practice. No use, distribution or reproduction is permitted which does not comply with these terms. 